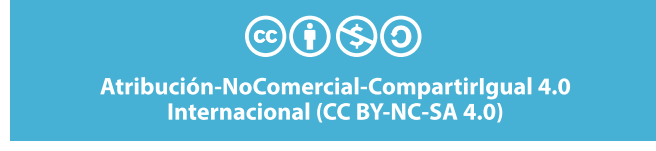

DOI: http://dx.doi.org/10.20983/reij.2022.1.3
Lázaro Rico Pérez ${ }^{1}$

Ivonne A. Gaytán Bertruy²

Guillermo Ramírez Armas ${ }^{3}$

Fecha de recepción: 8 de marzo de 2021

Fecha de aceptación: 8 de septiembre de 2021

\title{
JUSTICIA ALTERNATIVA: UNA EXPLICACIÓN DE SU COMPLEJIDAD A TRAVÉS DEL DISEÑO AXIOMÁTICO
}

\author{
Alternative justice and conflict complexity: an approach by axiomatic \\ design methodology
}

\section{Resumen}

En el presente trabajo, se presenta un ejemplo práctico relacionado con el derecho familiar para explicar la complejidad del conflicto social y presentar una solución al problema, a través de los mecanismos alternativos de resolución de controversias empleados en la justicia alternativa utilizando la metodología del diseño axiomático en las ciencias sociales. Los resultados muestran que mediante esta metodología, que tiene un sustento matemático, la complejidad de un problema disminuye si los recursos o alternativas de solución se incrementan. Por lo tanto, se concluye que por medio de la metodología del diseño axiomático se pueden resolver conflictos sociales, la cual ha sido validada en otras áreas del conocimiento.

Palabras clave: diseño axiomático; conflicto; mecanismos alternativos; complejidad.

\begin{abstract}
This paper presents a practical example related to family law to explain the complexity of social conflict and present a solution to the problem through alternative dispute resolution mechanisms used in alternative justice using the axiomatic design methodology in social science. The results show that through this methodology, which has a mathematical basis, the complexity of a problem decreases if the resources or alternative solutions increase. Therefore, it is concluded that through the axiomatic design methodology

1 Estudiante de Derecho de la Universidad Juárez Autónoma de Tabasco (UJAT). Correo electrónico: 173f28025@egresados.ujat. mx. ORCID: 0000-0003-0154-9073.

2 Profesora-investigadora de la Universidad Juárez Autónoma de Tabasco (UJAT). Correo electrónico: ivonne.gaytan@ujat.mx. ORCID: 0000-0002-8024-5168.

3 Profesor-investigador de la Universidad Juárez Autónoma de Tabasco (UJAT). Correo electrónico: grarmas41@gmail.com. ORCID: 000-0001-5155-4423.
\end{abstract}


social conflicts can be resolved which has been validated in other areas of knowledge.

Keywords: axiomatic design; conflict; alternative mechanisms; complexity.

\section{Introducción}

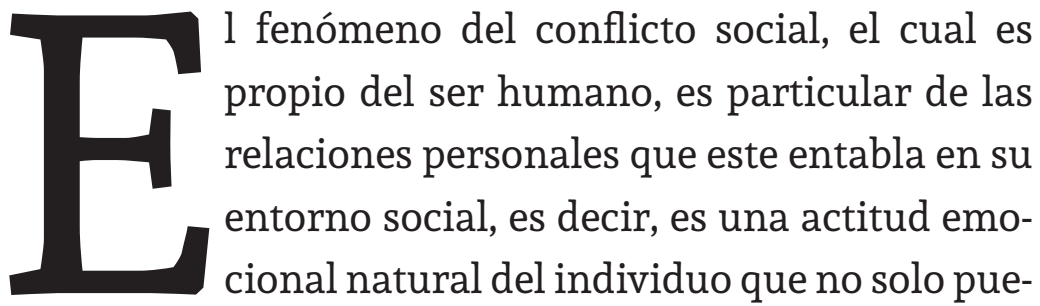
de ser negativa, sino también positiva, ya que ayuda a construir una cultura de paz (Carrillo-Pérez, 2016, p. 196). Además, se concibe como una parte de la vida diaria en la cual se buscan formas de solucionar los conflictos de manera pacífica sin llegar a la violencia; es una constante en la historia de la humanidad (Calderón Concha, 2009, p. 61). El conflicto en general nace como producto de la intolerancia a las diferentes formas de pensar del individuo, ya que este muchas veces cree que tiene la verdad absoluta e indiscutible, pero en las relaciones interpersonales la verdad absoluta no existe. Ahora, el conflicto se ve y surge en toda interacción social, por ejemplo, en la familia, entre padres e hijos, entre esposos, entre hermanos, en la escuela, entre maestro y alumno, entre alumnos, entre vecinos, en el trabajo, entre jefe-subordinado, entre trabajadores, etcétera. En resumen, donde exista discrepancia de pensamientos entre las partes nace el conflicto.

Ahora, en este fenómeno del conflicto, ¿cuál es la causa en particular del mismo? Muchos expertos han estudiado las causas del conflicto, sin embargo, este no ha sido estudiado desde una metodología que lo interprete desde el punto de vista axiomático, el cual ha sido some- 
tido rigurosamente a validez en diferentes campos, principalmente de la ingeniería, administración, entre otros, pero no ha sido usado y validado en el área de las ciencias sociales, específicamente en los conflictos sociales. Asimismo, La Rosa y Rivas (2018) lo sostienen y aclaran en su libro; no obstante, es necesario que tenga un sustento matemático como se lo da la metodología del diseño axiomático. Entonces, se plantea la siguiente hipótesis: Con la metodología del diseño axiomático, se pueden modelar los conflictos sociales relacionados con la justicia alternativa por la escasez de recursos.

Actualmente en la literatura se encuentra una vasta información respecto a la solución de conflictos: desde mecanismos radicalmente autocompositivos hasta heterocompositivos. También, otros expertos han estudiado las causas que originan el conflicto. Por ejemplo, hay estudios como el de La Rosa y Rivas (2018), quienes sostienen que las causas del conflicto pueden ser clasificadas de la siguiente manera:

- Deficiencia en la percepción de los hechos. Existe una percepción diferente de los hechos, que trae como consecuencia situaciones conflictivas. Desafortunadamente, muchas veces vemos el mundo con nuestro pensamiento y no tomamos una actitud empática de poder observar el mundo con ojos ajenos.
- Mala comunicación. Esta causa hace referencia a los malentendidos durante la comunicación, llevando al brote del conflicto entre las partes. La mayoría de las veces somos malos receptores, no sabemos escuchar con atención y solo nos interesa la comunicación en un solo sentido.

- Creencias distintas (valores). Este conflicto se origina por una creencia o pensamiento distintos entre personas; por ejemplo, algunas piensan que, en un matrimonio, el varón siempre tiene la razón, lo cual es una creencia machista. El no aceptar las creencias distintas es la semilla para la germinación de los conflictos, que solo pueden ser resueltos cuando aceptamos la amplia variedad de creencias y valores propios y ajenos.

- Estructura injusta. Se trata de escenarios en los cuales existen situaciones manifiestamente injustas; por ejemplo, una familia machista, un país con dictadura, etcétera.

- Recursos escasos. Son los casos en los que se disputa un bien escaso. Por ejemplo, cuando los bienes intestados que deja un difunto no alcanzan para, en igual proporción, darles a todos los hijos de la familia; el conflicto se hace más complejo de resolver conforme escasean los recursos. Cabe mencionar que la presente investigación, se centra en la resolución de conflictos cuando esto se origina por la escasez de recursos. La metodología del diseño 
axiomático será validada cuando un conflicto se origina por la escasez de recursos.

También La Rosa y Rivas (2018) se preguntaron: ¿cómo se resuelve un conflicto? Ellos sostienen que hay tres sistemas para resolver un conflicto:

- Los que se basan en el poder. Son aquellos donde se recurre a la fuerza física, institucional o económica, para poner fin a una situación conflictiva. Este sistema es autocompositivo, imponiéndose el más fuerte sobre el más débil.

- Los que se basan en el derecho. Estos métodos se basan en la aplicación de una norma, cuyos criterios objetivos ponen fin a un conflicto entre partes. En este sistema hay un ganador y un perdedor.

- Aquellos que se basan en intereses comunes que tienen las partes enfrentadas. Se pueden resolver a través de mecanismos comúnmente llamados alternativos para la resolución de conflictos, los cuales forman parte de la justicia alternativa mencionada en el artículo 17 constitucional. Estos mecanismos alternos serán detallados en los siguientes párrafos.

Los mecanismos alternativos de solución de conflictos (MASC) nacen a raíz de la deficiencia de los procesos judiciales, los cuales son los más arraigados en la sociedad y se caracterizan por ser tardíos y desgastantes de los estados emocionales de las partes involucradas en el conflicto; además, estos procesos se caracterizan por haber un ganador y un perdedor, y carecen de la voluntariedad de las partes, es decir, tienen carácter de obligatorio. Mas no así los MASC, que tienen carácter de voluntariedad, los cuales son procesos que requieren de un menor tiempo para alcanzar a resolver el conflicto, son económicos, buscan mantener las relaciones entre las partes a largo plazo, establecen un convenio en donde se detallan los acuerdos entre las partes y tienen como finalidad ganar-ganar por las partes en el conflicto. Se fundamentan en una cultura de paz y también se les llama justicia alternativa; en la actualidad existen centros de MASC públicos y privados. Los MASC públicos dependen del Tribunal Superior de Justicia de una entidad federativa, mientras que los MASC privados atienden al público siempre y cuando tengan la certificación emitida por el Estado para poder proporcionar servicio al público.

Recientemente, los MASC han tomado una importancia considerable en varios ámbitos del campo de las relaciones humanas, tales como el administrativo, deportivo, escolar, judicial, entre otros. Respecto a este último, el judicial, y de acuerdo con Azua (n. d.) y Cornelio (2014), el artículo 17 segundo párrafo de la Constitución Política mexicana sostiene que el individuo tiene el derecho de acceso a la justicia como derecho público y esta jus- 
ticia debe de ser impartida por tribunales expeditos dentro de los términos marcados por la ley. Asimismo, en el párrafo iv se menciona que para hacer más rápido los procesos judiciales, se dispone de los MASC, que son mecanismos alternos para resolver controversias. Esto es un derecho humano que la Carta Magna le da al individuo: el derecho a la justicia y sus modalidades.

Ahora, haciendo una breve historia de los MASC, en México, se puede decir que estos nacen a partir de 1993 con las negociaciones del Tratado de Libre Comercio, el cual le dio un impulso muy importante a su utilización. Así se menciona que los primeros usos que se les dio a los MASC fue para resolver conflictos comerciales; por lo tanto, los empresarios y abogados se dieron cuenta de la utilidad que estos tienen y lo rápido que se resuelve un conflicto de tipo comercial. Por otro lado, según Lobo Niembro (2019), en México ocurrió algo curioso respecto a las características centralistas en nuestro país: la incorporación de los MASC al marco constitucional fue de forma centrípeta, es decir, de afuera hacia el centro del país (la Federación); de entidades como Coahuila y Nuevo León hacia la Federación. Estos mecanismos de justicia alternativa han sido muy acogidos por los estados del país, ya que actualmente en cada entidad hay un centro o instituto de justicia alternativa. Además, estos centros o institutos ofrecen capacitación y formación para facilitadores. También, las universidades públicas en los diferentes estados han contribuido al fortalecimiento de la doctrina de los MASC. Tal es el caso de la Universidad Autónoma de Nuevo León, que tiene en sus programas de estudios la Maestría y Doctorado en Mecanismos Alternos de Solución de Conflictos; la Universidad Juárez Autónoma de Tabasco, que ofrece la Maestría y Doctorado en Métodos de Solución de Conflictos y Derechos; y la Universidad Autónoma de Nayarit, que ofrece la Maestría en Justicia Alternativa.

Es importante mencionar que el objetivo final de los mecanismos alternativos de solución de controversias es promover una cultura de paz, en donde las partes involucradas resuelvan sus controversias por voluntad propia y decidan por ellas mismas las alternativas de solución al conflicto en cuestión, sin necesidad de desgastarse emocionalmente en un juicio procesal en el cual el juez decidirá por ellas y habrá un ganador y un perdedor. Mas no así en los MASC, donde el objetivo es ganar-ganar por las partes involucradas. Aunque cabe mencionar que muchos abogados inicialmente estaban incrédulos de que un conflicto se podía llegar a resolver por estos medios, es importante mencionar que con estos se reduce la cantidad de expedientes esperando turno, esos gruesos expedientes; no así en los mecanismos alternativos, que en un ambiente de armonía buscan 
resolver o "destrabar" el conflicto que impide la convivencia pacífica entre las partes.

Estos MASC son muchas veces de carácter preventivo, es decir, pueden ser de ayuda para prevenir un delito de mayores consecuencias; por ejemplo, en un caso de acoso escolar, en el cual se puede recurrir al MASC llamado mediación para resolver problemas de tipo escolar. Este tipo de conflictos se puede mediar llegando a resultados adecuados para resolver el conflicto antes de que el problema trascienda hasta los tutores de los alumnos, que pueden en un determinado momento llegar a un enfrentamiento e incurrir en daños contra su persona o su patrimonio.

Entre los métodos que emplean los MASC para resolver conflictos están la negociación, mediación, conciliación y arbitraje, cada uno de ellos con sus respectivas características. Estos MASC tienen una característica particular: son autocompositivos, es decir, dan soluciones acordes a las necesidades fuera de un proceso contencioso. Solo el arbitraje es un mecanismo alternativo de tipo heterocompositivo, en el cual es necesario un árbitro, una persona ajena que decida por las partes. Algunos expertos sostienen que el arbitraje es la forma más común de resolver las controversias en Occidente.

Los MASC tienen dos características que los hacen útiles respecto a los litigios: la rapidez y la eficacia, tal y como lo men- ciona Santamaría (2007), quien, además, sostiene que fomentan la paz, el trabajo participativo, la tolerancia, la empatía, el diálogo, entre otros, para encontrar soluciones que lleven a resultados lo más aproximados al ganar-ganar de las partes. Asimismo, se fundamentan en un grupo de principios, como voluntariedad, confidencialidad, flexibilidad, simplicidad, imparcialidad, igualdad, honestidad, independencia y neutralidad (Gorjón Gómez, Adame, \& Salazar, 2018, p. 26). En los siguientes párrafos, se discutirán brevemente los principios antes mencionados.

\section{Voluntariedad}

Es de los principios más importantes en los que se fundamentan los MASC. Para que se lleve a cabo la solución de la controversia de las partes, debe existir una voluntariedad de ambas partes, libre de obligación y coacción. A diferencia de los procesos judiciales no es obligatorio aceptar el apoyo de los MASC. Este es resultado de la voluntad de ambas partes.

\section{Confidencialidad}

Es otro de los principios importantes dentro de los MASC, el cual involucra a las partes, a los asesores legales, y los compromete a la no-divulgación de todo lo acordado durante el intercambio de ideas en las reuniones. Muchas veces las partes quieren mantener privada su vida y sus problemas o conflictos; por lo tanto, quie- 
ren que se les garantice privacidad total en la información vertida durante el proceso de resolución del conflicto. Bajo este principio, se puede garantizar a las partes una total confidencialidad por parte de los mediadores.

\section{Flexibilidad}

Los MASC no se sujetarán a normas estrictas o dogmas o "trabas”. Estos mantienen cierta flexibilidad respecto a fechas, horarios y formas de intervenir durante las reuniones. No se imponen formalidades ni solemnidades innecesarias.

Así, el facilitador debe de ser amable pero firme; la flexibilidad no significa que debe de haber un relajamiento de los procedimientos empleados en los MASC. El facilitador debe de ser asertivo, empático y buscar la forma de motivar a las partes para participar y generar ideas para llegar a una pronta resolución del conflicto.

\section{Simplicidad}

Los procedimientos de justicia alternativa deberán ser sencillos, evitando todo tipo de formalidades y solemnidades innecesarias. Los procedimientos utilizados deben ser parsimoniosos. Se debe de evitar la imposición de reglas que hagan el procedimiento lento, burocrático y engorroso. En pocas palabras: se tienen que eliminar las formas innecesarias que demoren el proceso de resolución del conflicto.
Imparcialidad

El facilitador no debe de esperar que todos piensen como él. Somos seres humanos, pensamos diferente y es importante mantener una postura de imparcialidad, alejada de todo sesgo religioso, ideología política, etcétera. Debe mantener una mente imparcial y objetiva, para que las partes en controversia se sientan en confianza suficiente y puedan expresar sus ideas e inconformidades libremente.

\section{Equidad o igualdad}

Debe verse como un sistema de contrapesos. Este principio supone otra obligación del facilitador que debe de usar sus estrategias para conducirse con equidad en el trato con las partes. Así, por ejemplo, una de las partes puede ser un doctor en derecho y la otra puede ser una persona sin estudios; sin embargo, el facilitador que se conduce con equidad debe tratar por igual a ambas personas, dirigirse a ellas como señor Pérez, señor García, y no doctor Pérez, porque se pierde la equidad en el trato con las personas que quieren resolver la controversia.

\section{Honestidad}

Se espera del facilitador que se conduzca con toda la honestidad debida, con la rectitud necesaria para conducirse bien en el proceso de resolución de controversias. El facilitador debe mantener la balanza entre las partes; que no se noten intenciones 
de sesgo o que se incline la balanza hacia alguna de ellas, porque esto solo indicará una falta de honestidad por parte del facilitador.

Después de hablar de los principios de los MASC, ahora se tratarán sus ventajas o cuál es la diferencia que los hace una mejor alternativa que un proceso adversarial. Gorjón Gómez y Steele Garza (2016) hicieron una clasificación taxativa de características que hacen usar los MASC sobre el proceso adversarial.

\section{Amplitud de la solución}

De acuerdo con Gorjón Gómez y Steele Garza (2016), el litigio solo trata aspectos puramente legales; sin embargo, a través de los MASC la solución al conflicto puede estar relacionada con aspectos que van más allá de solo asuntos legales; por ejemplo, aspectos emocionales, de comunicación, de acercamiento de las partes, que pueden ser tratados en un ambiente armonioso sin pensar en un contraataque, tal y como se presenta en un proceso adversarial (Carrillo-Pérez, 2016).

\section{Mantenimiento de relaciones futuras}

Dado que los MASC operan dentro de un ambiente armonioso, donde el Principio de Voluntariedad nace en cada una de las partes ávidas de solucionar en forma pacífica un conflicto, en donde predomina la meta de ganar-ganar, la probabilidad de mantener las relaciones a futuro es alta. Mas no así en el proceso adversarial, el cual se fundamenta en el contraataque, con las intenciones de ganar-perder en ambas partes, es decir, siempre habrá un perdedor; por lo tanto, las relaciones se fracturan a largo plazo.

\section{Celeridad}

Dado que el proceso adversarial es desgastante, tanto para las partes involucradas como para sus familiares, ya que consume muchas horas, etcétera, la solución de conflictos entre las partes mediante los MASC es más rápida, por lo que se evitan muchos sufrimientos personales y familiares de ambas partes en el conflicto.

\section{Resultados permanentes}

Los acuerdos logrados por las partes involucradas son permanentes, es decir, las partes mantienen sus relaciones a largo plazo en condiciones de paz y armonía.

\section{Economía}

Los recursos económicos invertidos en la resolución de un conflicto social, a través de los MASC, son mínimos; con ello, se protege el aspecto económico de ambas partes y sus familias.

\section{MASC y sus características}

A continuación, se discutirán brevemente los diferentes MASC, así como sus características. 


\section{Negociación}

Es el proceso en el que dos o más partes que quieren resolver un conflicto, a través de logros y objetivos, emplean diversas técnicas de comunicación para alcanzar sus fines de una manera armoniosa y pacífica.

Una técnica muy recurrida en la negociación es el método Harvard, que está conformado por seis etapas:

- Opciones: de acuerdo con Gorjón Gómez et al. (2018) son todas las expectativas que las partes pueden tener una vez que han dejado a un lado sus posiciones y comprenden y entienden las pretensiones de la parte contraria.

- Alternativas: son las opciones de las partes; entre más alternativas existan la probabilidad de resolución del conflicto es más alta.

- Legitimación: consiste en lo justo y lo equitativo de lo que se pretende con las soluciones propuestas.

- Comunicación: tal y como expresan Gómez Duarte y González López (2020):

Un hombre aislado; es un hombre que no muestra sentido. El individuo es naturalmente relacional y por lo tanto generador de conflictos. Esta etapa es de las más importante parar llegar a una buena resolución del conflicto. Una comunicación de ambas partes, fluida, armónica es lo deseable (p. 126).
- Relaciones: se busca mantener una sólida relación entre las partes por mucho tiempo.

- Acuerdos: la etapa final de este método es lograr acuerdos que dejen satisfechas a ambas partes; es decir, debe de ser un acuerdo manifestado, a través de un convenio, cuya finalidad es la de ganar-ganar.

\section{Características}

- Alcanzar un clima armonioso de comunicación para lograr beneficios comunes de ambas partes.

- No hay límite de personas y tampoco en materia judicial.

- Las técnicas de comunicación pueden ser variadas.

\section{Mediación}

Las partes resuelven un conflicto mediado por un tercero. Este método es de los más empleados en mediación familiar, civil, escolar, entre otras (Gorjón Gómez, \& Steele Garza, 2016).

\section{Características}

- Las partes son guiadas por un tercero y solas solucionan el conflicto; es decir, el tercero solo guía a las partes, pero no interviene en la solución del conflicto.

- El tercero debe de ser un experto en la materia. 
- El proceso debe de terminar en el momento en que alguna de las partes lo decidan.

- No hay ganadores ni perdedores.

- Las partes designan el lugar y hasta el idioma del proceso.

- Es muy rápido y voluntario.

- Los resultados son voluntarios.

Campos de aplicación de la técnica de mediación para resolver conflictos

\section{Mediación familiar}

Se ejecuta con éxito en otros países como Estados Unidos y Argentina. Este método tiene como finalidad la humanización de las partes involucradas en el conflicto. Este instrumento tratará de resolver amistosamente los conflictos que puedan surgir en la familia con apoyo de un mediador, quien solamente guiará a las partes en un ambiente armonioso para que puedan llegar a una solución.

Para que se pueda llevar a cabo el proceso de resolución de conflictos mediante el método de mediación, debe de existir la voluntad de ambas partes involucradas. Es un proceso voluntario que se puede terminar en el momento en que cualquiera de las partes lo decida. Los tipos de conflictos a tratar son variados dentro de la mediación familiar. A continuación se discuten algunos, según Gorjón Gómez y Steele Garza (2016). Cabe mencionar que el fin último de la mediación familiar es el bienestar, primeramente de los hijos, quienes son ajenos a los problemas de los mayores y después los demás miembros de la familia.

\section{Tipos de problemas familiares}

- Derecho de alimentos: que los niños y otros miembros de la familia cuenten con los medios para satisfacer sus necesidades.

- Derecho de convivencia cuando hay padres separados: la convivencia familiar entre padres e hijos cuando estos están separados.

- Derecho de cuidado y crianza: que los niños vivan con el padre más apto psicológica, física y económicamente; que se garantice la salud emocional y física del hijo o los hijos.

- Separación de bienes: alcanzar una separación de bienes equitativa; siempre cuidando de que el uso de los bienes sea en beneficio primordialmente de los hijos menores de edad.

Entre estos bienes se pueden mencionar los siguientes:

Bienes inmuebles

Cuentas bancarias

Vehículos

Joyas

Pieles, objetos de arte, antigüedades, etcétera. 
Conflictos sucesorios: mantener la unión familiar durante la etapa sucesoria; que la repartición de bienes no sea motivo de conflictos familiares.

Cuidado de miembros de la familia: hay que asegurar que la familia cumpla su función de protectora de los hijos menores de edad.

Etapas de la mediación

Según Haynes, citado por Gorjón Gómez y Steele Garza (2016), las etapas de la mediación son:

Se detecta el problema que será el punto de discusión por ambas partes.

Se escoge al mediador con ciertas características importantes.

Se recopila la información para tener una idea de la situación problemática.

Se redefine el problema.

Se generan opciones de solución.

Se redefinen las posturas.

Se empieza la negociación.

Se redactan acuerdos.

Características del mediador

El mediador no debe de tener precisamente estudios de psicología, pero sí debe de tener algunas características importantes, para que pueda desempeñar su trabajo adecuadamente.

Capacidad para motivar.

Habilidad para generar soluciones.
Ser una persona de mente abierta.

Flexibilidad en las ideas.

Ser empático.

Capacidad de liderazgo.

Que sea totalmente imparcial.

Finalmente, si el mediador es un profesional se recomienda que sea del área de ciencias de las humanidades.

\section{Mediación penal}

Respecto a la mediación penal pasa algo curioso: este método tiene mejor efectividad antes de que el delito se materialice. Por ejemplo, en el caso de dos vecinos uno de ellos molesta al otro con su mascota cuando esta hace sus necesidades fisiológicas en su patio. Si ambos vecinos solicitan la ayuda de un mediador para resolver el conflicto, se podrá volver armonizar la relación entre ambos. Con estas acciones, se puede llegar a evitar que se materialice el delito ocasionado por conductas y estados irracionales.

\section{Conciliación}

Es el método en el cual las partes nombran a un tercero, para que, a través de él, se puedan poner de acuerdo para resolver el o los conflictos en común.

\section{Características}

- El tercero propone la solución a las partes y emplea su capacidad de persuasión, para que estas acepten su 
propuesta (esta característica la hace diferente a la mediación. Es decir, el conciliador propone y persuade a las partes para que acepten su propuesta de solución, mientras que el mediador solo conduce de forma armoniosa las pláticas entre las partes sin proponer alguna solución).

- El conciliador debe de ser un experto en la materia.

- Esta técnica le antecede al arbitraje.

- El conciliador formula un informe.

- El cumplimiento de la técnica debe de ser voluntario.

\section{Arbitraje}

Es un método heterocompositivo extraprocesal, que se fundamenta en la voluntariedad de las partes y en donde un tercero, llamado árbitro, decide por ellas. Es muy utilizado en el comercio internacional, además de que es considerado el mejor método de solución de conflictos en dicho ámbito. El origen del arbitraje es eminentemente contractual, de modo que su validez y operatividad dependen en todo momento del cumplimiento del pacto acordado entre las partes.

El arbitraje se distingue de los demás MASC por su formalidad; un árbitro decide por las partes, que le ceden su potestad y es quien le da una solución al conflicto. Pero cabe aclarar que, aunque el arbitraje es un mecanismo formal, no se está diciendo que los otros mecanismos carez- can de esta formalidad. Su parte esencial es el acuerdo, el cual es vinculatorio. Este acuerdo arbitral puede adoptar la forma de una cláusula compromisoria o acuerdos independientes (Quintanilla, s.f.).

\section{Características del árbitro}

Las características de un buen arbitraje, se reducen a tres principales:

- Flexibilidad

- Rapidez

- Inmediatez

Ahora, como se mencionaba en párrafos anteriores, los MASC han tenido un amplio campo de aplicación dentro de las relaciones interpersonales en las cuales se generan una gran variedad de conflictos. Así, por ejemplo, Lozano (2018) en su tesis doctoral muestra la utilidad que tienen los MASC dentro de la psicología en el deporte. Sus resultados evidencian que la utilización de los MASC, especialmente la mediación, puede contribuir a generar un ambiente de armonía, reducir la tensión entre los jugadores, etcétera.

Por otro lado, Esquivel (2015) en su tesis doctoral muestra la utilidad de los MASC en el campo educativo. En su trabajo evidencia la importancia de fomentar una cultura de paz dentro del ámbito educativo; exaltar y fomentar los valores entre la comunidad académica. Finalmente, De la Rosa (2017) muestra la utilidad de los 
MASC en los conflictos matrimoniales; en sus resultados menciona que resolver problemas matrimoniales mediante los MASC tiene las ventajas siguientes: las soluciones son rápidas y económicas, y las relaciones no se desgastan con litigios largos.

Ahora, los MASC son usados para resolver conflictos y estos, por su naturaleza, son complejos, porque involucran al comportamiento humano con una amplia variedad de atributos que forman su personalidad particular, como estados de coraje, tristeza, dolor físico, entre otros más. Asimismo, involucran capacidades cognitivas derivadas muchas veces de su entorno social (Reza et al., p. 125). Esta complejidad de los conflictos hace más difícil la aplicación de los MASC en la justicia alternativa. En la literatura, se encuentra información relacionada con la Teoría del Conflicto y su relación y resolución con los MASC; sin embargo, es un análisis muy superficial. Tal es el caso de Cabello Tijerina (2018), quien propone un mapeo para la resolución de un conflicto. Por lo tanto, es importante entender la complejidad del conflicto para después llevarlo a la aplicación de los MASC.

\section{Metodología del diseño axiomático}

El diseño axiomático es una metodología para diseñar sistemas complejos, que fue propuesta en el Instituto Tecnológico de Massachusetts por Nam Pyo Suh en 1970
(Dana et al., 2021, p. 244). El diseño axiomático tuvo sus aplicaciones iniciales en el área de diseño del producto y, además, ha sido validado en muchas áreas del conocimiento. Así, por ejemplo, Dana et al. (2021) usaron el diseño axiomático para optimizar la puerta de un robot móvil y sus resultados mostraron un diseño óptimo de los parámetros de funcionalidad de la puerta del robot, a través de sus requerimientos funcionales. Por otro lado, Liu et al. (2018), mediante la metodología del diseño axiomático, diseñaron el proceso de construcción de un vehículo militar con toda la complejidad de tareas relacionadas con el mismo.

Una revisión de la literatura fue hecha por Rauch, Matt y Dallasega (2016) acerca de la aplicación de la metodología del diseño axiomático en los sistemas de manufactura. También, el diseño axiomático ha tenido aplicación en la industria de servicios. Tal es el caso de Peck y Sang-Gook (2010), quienes emplearon la metodología del diseño axiomático para mejorar el flujo de pacientes atendidos en el Departamento de Emergencias de un hospital. Los resultados mostraron un decremento del cincuenta por ciento en el tiempo de espera por cada paciente. Asimismo, Giang y Shenoy (2005) propusieron una teoría utilitaria para la toma de decisiones bajo incertidumbre. Los resultados muestran que el diseño axiomático es efectivo para desarrollar una teoría adecuada para la 
toma de decisiones. Albano y Suh (1994) propusieron usar la metodología del diseño axiomático para estructurar, a través de la ingeniería concurrente, el desarrollo de un producto. Finalmente, Arcidiacono, Giorgetti y Pugliese (2015) aplicaron el diseño axiomático para conectar los requerimientos funcionales o necesidades del cliente al trasladarse con todos los elementos del proceso y sus limitaciones, como los aspectos de infraestructura, los límites impuestos por seguridad y la disponibilidad de seguridad y equipo. En sus resultados recomiendan algunos aspectos de mejoramiento para optimizar el pasaje, a través de la terminal, y asegurar una completa accesibilidad de los ambientes considerados. En esta investigación será validada el área del derecho, específicamente la justicia alternativa en la resolución de conflictos.

Ahora, se explicará brevemente la metodología del diseño axiomático. Según Suh, citado por Gonçalves-Coelho y Mourão (2007), este se estructura a través del mapeo de cuatro dominios; quedando como se muestra en la figura 1 :
Figura 1. Estructura del diseño axiomático

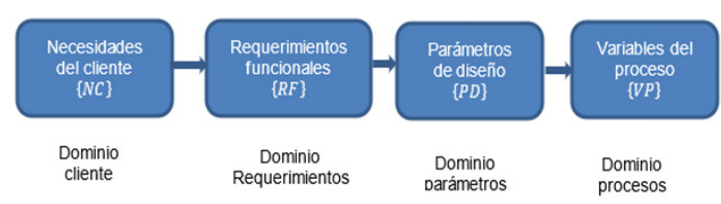

Fuente: Suh, citado por Gonçalves-Coelho y Mourão (2007).

El diseño axiomático se fundamenta en dos axiomas:

El Axioma de Independencia, que enuncia que los requerimientos funcionales se deben satisfacer sin afectar a ningún otro requerimiento.

El Axioma de Información, que sostiene que la mínima información contenida en el sistema da la mejor solución para el diseño.

Matemáticamente, el Axioma de Independencia queda representado como:

$$
\{R F\}=\{A\}\{P D\}
$$

Donde:

$\mathrm{A}$ = Matriz de diseño; siendo A una matriz identidad, que permite mantener la independencia entre los requerimientos funcionales:

$$
A=\left[\begin{array}{ll}
1 & 0 \\
0 & 1
\end{array}\right]
$$

Por otro lado, el Axioma de Información se expresa matemáticamente como: 


$$
I=\log _{x} \frac{1}{P}
$$

Donde:

$\mathrm{I}=$ Cantidad de información contenida en la solución de un diseño; se mide en bits

$\mathrm{P}=$ Probabilidad de alcanzar el objetivo de un requerimiento funcional (rf)

Ahora, el rango común en el cual se mide la probabilidad de éxito en una solución específica de diseño es:

$$
P=\int_{c r 1}^{c r 2} d R F
$$

Validación de la metodología axiomática para resolver un conflicto cuando hay escasez de recursos, a través de un ejemplo práctico ficticio

Para validar la utilidad que tiene la metodología del diseño axiomático para resolver conflictos sociales cuando existe escasez de recursos, usaremos un caso práctico ficticio. En el caso del conflicto, analizándolo desde el diseño axiomático, este se complica conforme el número de recursos o alternativas de solución disponibles para resolver el conflicto disminuye. Es decir, la solución del conflicto está relacionada con los recursos existentes. Esto también lo dicen La Rosa y Rivas (2018), quienes mencionan que una de las causas que genera el conflicto social es la escasez de alternativas de solución; ahora se demostrará matemáticamente esta aseveración, a través de la metodología del diseño axiomático. Para demostrarlo, se empleará el siguiente ejemplo práctico: en un litigio familiar los representantes de la justicia alternativa pretenden salvar un matrimonio que presenta conflictos económicos, lo cual los ha llevado a una decisión de divorcio. Hay dos pretensiones de los litigantes o partes en conflicto: por un lado, que se generen más recursos económicos, y por el otro, buscar formas de ahorros significativos en el hogar. Por lo tanto, usando la metodología del diseño axiomático se tiene el vector de pretensiones, el cual queda como sigue:

$$
\{P T\}=\left\{\begin{array}{l}
P T 1 \\
P T 2
\end{array}\right\}
$$

Donde:

$\{\mathrm{PT}\}=$ Vector de pretensiones de los litigantes o partes para quedar satisfechos y evitar un divorcio

$\{$ PT1 $\}=$ Generar más recursos económicos para el hogar

$\{$ PT2 $\}=$ Ahorro en los gastos del hogar

El vector recursos o alternativas está expresado por el vector:

$$
\{R\}=\left\{\begin{array}{l}
R 1 \\
R 2 \\
R 3 \\
R 4
\end{array}\right\}
$$

Donde:

\{ R1 $\}=$ Uno de los cónyuges busca un trabajo para generar más recursos económicos para el hogar 
\{ R2 $\}=$ Minimizar los gastos de luz eléctrica comprando focos ahorrativos, usando la refrigeración solo cuando se requiera (en tiempos de temperaturas muy altas, como en el verano)

\{ R3 \} = Inscribir a hijos en escuelas públicas y no privadas para reducir gastos

$\{\mathrm{R} 4\}=$ Mudarse a una casa con una renta mensual más baja que la actual

Ahora, aplicando la metodología del diseño axiomático, a través del Axioma de Independencia, se tiene:

$$
\{P T\}=\{A\}\{R\}
$$

Ahora, sustituyendo las ecuaciones (5) y (6) en (7), se tiene:

$$
\left\{\begin{array}{l}
P T 1 \\
P T 2
\end{array}\right\}=\{A\}\left\{\begin{array}{l}
R 1 \\
R 2 \\
R 3 \\
R 4
\end{array}\right\}
$$

La matriz de diseño puede quedar como:

$$
\left\{\begin{array}{l}
P T 1 \\
P T 2
\end{array}\right\}=\left[\begin{array}{llll}
1 & 0 & 0 & 1 \\
0 & 1 & 1 & 0
\end{array}\right]\left\{\begin{array}{l}
R 1 \\
R 2 \\
R 3 \\
R 4
\end{array}\right\}
$$

En este caso, se puede notar que la solución es matemáticamente redundante, debido a que hay más alternativas o recursos que pretensiones, logrando con esto que el conflicto sea más sencillo de resolver; es decir, existen más alternativas de solución que pretensiones. En general, un conflicto se vuelve más complejo cuando los recursos o alternativas que se tienen son menores a las pretensiones, tal y como lo vemos en el siguiente ejemplo:

Siguiendo con el ejemplo inicial, las pretensiones siguen siendo dos, pero ahora los recursos y alternativas de solución solo son una. Entonces, se tiene:

$$
\begin{aligned}
& \left\{\begin{array}{l}
P T 1 \\
P T 2
\end{array}\right\}=\{A\}\{R 1\} \\
& \left\{\begin{array}{l}
P T 1 \\
P T 2
\end{array}\right\}=\left[\begin{array}{l}
1 \\
0
\end{array}\right]\{R 1\}
\end{aligned}
$$

En este ejemplo modificado solo se satisface la pretensión PT; sin embargo, la pretensión PT no se satisface por falta de recursos o alternativas. Entonces, de manera general, el conflicto tiende a la complejidad entre menos recursos o alternativas de solución se tengan para resolverlo. Entre más recursos o alternativas de solución se generen, la complejidad del conflicto disminuye y la probabilidad de lograr el objetivo de acuerdos en un ambiente de paz será más alta. Ahora, hablando con palabras del Axioma de Información, se puede decir que si la información que se dispone para resolver un conflicto aumenta, la probabilidad de éxito se incrementa.

\section{Conclusiones}

Con la metodología del diseño axiomático, que es una metodología validada en varios 
campos de la ingeniería y la administración, se explicó el conflicto social y su entendimiento dentro de la justicia alternativa. Mediante los dos axiomas del diseño axiomático, se mostró que el conflicto entre las partes es una cuestión de recursos o alternativas disponibles para resolverlo. Además, es una forma sistémica de demostrar que el conflicto está relacionado con la escasez de recursos, es decir, un conflicto se agrava entre menos alternativas de solución existan. Por lo tanto, se concluye a través de la metodología del diseño axiomático, la cual está rigurosamente validada en otros campos de la ciencia, pero poco o nada explorada en las ciencias sociales, a través de un ejemplo práctico, que un conflicto aumenta su complejidad si sus alternativas de solución son pocas. Esto lo mencionan La Rosa y Rivas (2018) como la falta de recursos disponibles o alternativas de solución como generador de conflictos, pero, ahora, con esta investigación y mediante el diseño axiomático, se demuestra matemáticamente por qué la escasez de recursos genera conflictos sociales entre las partes. Ahora, la hipótesis planteada inicialmente se corrobora, es decir, la metodología del diseño axiomático es útil para modelar conflictos sociales cuando existe escasez de recursos. Finalmente, se recomienda para trabajos futuros continuar utilizando técnicas o metodologías que son propias de la ingeniería y otras ciencias para aplicarlas en el área de las ciencias sociales.

\section{Referencias}

Albano, L. D., \& Suh, N. P. (1994). Axiomatic Design and Concurrent Engineering. Comp.Aid. Des., 26(7), 499-504.

Arcidiacono, G., Giorgetti, A., \& Pugliese, M. (2015). Axiomatic Design to Improve PRM Airport Assistance. Procedia CIRP, 34, 106-111. Ariza Santamaría, R. (2007). Estado del arte de los mecanismos de resolución de conflictos en colombia. IUSTA, 58-73.

Azua Aleman, E. (S.F). Mecanismos alternativos de solución de controversias en el juicio Agrario. THEMIS, 37-42.

Cabello Tijerina, P. A. (2018). Theory and Conflict Management. In: F. J. Gorjón Gómez, \& R. Chávez de los Ríos, Manual de mediación penal, civil, familiar y justicia restaurativa (pp. 59-68). Tirant lo Blanch.

Calderón Concha, P. (2009). Teoría de conflictos de Johan Galtung. Rev. Paz Conf., 2, 60-81. Carrillo-Pérez, R. (2016). Resolución de conflictos: hacia una cultura de paz en niños de primaria. Ra Ximhai, 12(3), 195-205.

Clauer, D., Fottner, J., Rauch, E., \& Prüglmeier, M. (2021). Usage of Autonomous Mobile Robots Outdoors-An Axiomatic Design Approach. Procedia CIRP, 96, 242-247.

Cornelio Landero, E. (2014). Los mecanismos alternativos de solución de controversias como derecho humano. Barataria, 81-95. 
De la Rosa Vazquez, C. (2017). Construcción de la cultura de paz. Monterrey: Universidad Autónoma de Nuevo Leon.

Erwin, R., Matt, D. T., \& Dallasega, P. (2016). Application of Axiomatic Design in Manufacturing System Design: A Literature Review. Procedia CIRP, 53, 1-7.

Esquivel Marín, C. (2015). Prácticas restaurativas y mediación escolar: medidas alternativas para la prevención y solución de conflictos escolares. Monterrey: Universidad Autónoma de Nuevo Leon.

García, J. V. (s.f.). Mediación familiar (M. L. Garza, entrevistador).

Giang, P. H., \& Shenoy, P. P. (2005). Two Axiomatic Approaches to Decision Making using Possibility Theory. Europ. J. Operat. Res., 162(2), 450-467.

Gómez Duarte, M. P., \& González López, L. E. (2020). Agentes de paz: la potencia generativa de lo cotidiano. Rev. Paz Conf., 13(1), 125148.

Gonçalves-Coelho, A. M., \& Mourão, A. J. F. (2007). Axiomatic Design as Support for Decision-making in a Design for Manufacturing Context: A Case Study. Int. J. Prod. Econ., 109(1-2), 81-89.

Gorjón Gómez, F. J., Adame, M., \& Salazar, R. (2018). Mediation and Alternative Dispute Settlement Mechanisms: Definition, Principles and Characteristics. In: F. J. Gorjón Gómez, \& R. Chávez de los Ríos, Criminal Civil, Family and Restorative Justice Mediation Handbook (pp. 21-28). Tirant lo Blanch.
Gorjón Gómez, F. J., \& Steele Garza, J. G. (2016). Métodos alternativos de solución de conflictos. Oxford.

La Rosa, J., \& Rivas, G. (2018). Teoría del conflicto y mecanismos de solución. Fondo Editorial.

Liu, W., Ji, J., Yang, Y., \& Zhang, L. (2018). Capability-based Design Task Decomposition in Heavy Military Vehicle Collaborative Development Process. Procedia CIRP, 70, 13-18.

Lobo Niembro, R. (2019). Mecanismos alternos de solución de controversias. Tirant Lo Blanch. Lozano, J. M. (2018). La psicología en el deporte y su impacto en la resolución de conflictos a través de la mediación. Universidad Autónoma de Nuevo León.

Peck, J., \& Sang-Gook K. (2010). Improving Patient Flow through Axiomatic Design of Hospital Emergency Departments. CIRP J. Manuf. Sci. Technol., 255-260.

Quintanilla, G. M. (s.f.). Poder Judicial del Estado de Nuevo León. http://www.pjenl.gob. $\mathrm{mx} /$ ConsejoJudicatura/CEMASC/Articulos/ arbitraje.pdf

Reza, S., Mohammad, A., Ali, A. T., \& Mina, T. (2015). Dynamic Axiomatic Design(): Applying the Independence Axiom in the Design of Social Systems. Procedia CIRP, 34, 125-130. Vladimir, M., \& Slavomir, B. (2015). Using Axiomatic Design and Entropy to Measure Complexity in Mass. Procedia CIRP, 87-92. 